\title{
Quais as estratégias de ventilação pulmonar na síndrome do desconforto respiratório agudo causada pela COVID-19? Um estudo de revisão
}

What are the pulmonary ventilation strategies in the acute respiratory distress syndrome caused by COVID-19? A review study

Cuáles son las enfermedades pulmonares en el síndrome de dificultad respiratoria aguda causado por COVID-19? In estudio de revisión

Maria Itamara da Silva Oliveira

ORCID: https://orcid.org/0000-0002-5022-2862 Universidade Federal da Paraíba, Brasil E-mail: itamara.oliveiraa@gmail.com

Celso Brendo Furtado Brandão

ORCID: https://orcid.org/0000-0001-8591-794X Universidade Federal da Paraíba, Brasil E-mail: celso.brendo@gmail.com

Karina Vieira da Costa

ORCID: https://orcid.org/0000-0002-6225-6373 Universidade Federal da Paraíba, Brasil E-mail: karinavcosta16@gmail.com

Joyce Polàine dos Santos Silva

ORCID: https://orcid.org/0000-0002-3053-3516 Universidade Federal da Paraíba, Brasil E-mail: joycepolaine1@gmail.com

Vanessa Michelle de Souza Fernandes ORCID: https://orcid.org/0000-0002-6360-7972 Universidade Federal da Paraíba, Brasil E-mail: vanessamichellefisio@gmail.com

Geraldo Eduardo Guedes de Brito ORCID: https://orcid.org/0000-0002-3059-3164 Universidade Federal da Paraíba, Brazil E-mail: eduardo.guedes.ufpb@gmail.com

Ubiracé Fernando Elihimas Júnior ORCID: https://orcid.org/0000-0001-5426-4253 Universidade de Pernambuco, Brazil E-mail: ubirace.elihimas@ieprhp.org.br

Eduardo Eriko Tenório de França ORCID: https://orcid.org/0000-0001-9207-2180 Universidade Federal da Paraíba, Brazil E-mail: edueriko@hotmail.com

\begin{abstract}
Resumo
No final de 2019, emergiu um grupo de casos de pneumonia associados a um novo coronavírus de RNA envelopado, espalhando-se rapidamente pelos continentes e sendo rotulado como pandemia global por COVID-19. Uma proporção considerável da população doente não necessitará de hospitalização, entretanto, alguns pacientes podem desenvolver a forma grave da doença e apresentar complicações como a síndrome do desconforto respiratório agudo (SDRA), requerendo ventilação mecânica invasiva (VMI). Pacientes com SDRA por COVID-19 podem apresentar algumas características específicas em comparação com as formas típicas da síndrome, desenvolvendo fenótipos distintos que ainda tem sido estudados. As pesquisas têm, então, se concentrado em esclarecer estratégias ventilatórias protetoras do pulmão (EPP) para estes pacientes. Esta revisão narrativa teve como objetivo investigar a experiência mundial atual em relação à EPP como manejo da SDRA associada à COVID-19 e destacar considerações para as práticas de VMI durante a pandemia. Após um processo de rastreamento de estudos nas bases de dados PubMed e BVS, foram selecionados 20 estudos a serem analisados. Na maior parte dos estudos o manejo ventilatório dos pacientes se assemelhou ao da SDRA "clássica” e, portanto, a EPP destacou-se como importante aliada na regressão da SDRA nos pacientes COVID-19. No entanto, deve ser levado em consideração a heterogeneidade fenotípica dos pacientes, principalmente quanto à mecânica pulmonar e capacidade de recrutamento. Este estudo possibilitou aprofundamento
\end{abstract}


cientifico sobre as semelhanças e diferenças da SDRA por COVID-19 e por outras causas, além de permitir a formação de orientações quanto aos ajustes ventilatórios iniciais em pacientes com SDRA/COVID-19.

Palavras-chave: COVID-19; Betacoronavirus; Respiração artificial; Síndrome do desconforto respiratório do adulto.

\begin{abstract}
In late 2019, a group of pneumonia cases associated with a new enveloped RNA coronavirus, spreading rapidly across continents and being known as the global COVID-19 pandemic. A smaller part of the population will not require hospitalization, however, some patients may develop a severe form of the disease and present complications such as an acute respiratory distress syndrome (ARDS) that requires invasive mechanical ventilation (IMV). Patients with ARDS by COVID-19 may present some specific characteristics in comparison with the typical forms of the syndrome, developing distinct phenotypes that have still been studied. Research has focused on clarifying protective lung ventilation (PLV) for these patients. This narrative review aimed to investigate the current worldwide experience in relation to PLV as management of ARDS associated with COVID-19 and to highlight IMV practices during a pandemic. After a process of tracking studies in the PubMed and BVS databases, 20 studies were selected. In most studies, the ventilatory management of patients is similar to the "classic" ARDS and, therefore, an PLV stood out as an important ally in the regression of ARDS in COVID-19 patients. However, the patients' phenotypic heterogeneity must be taken into account, especially regarding pulmonary mechanics and recruitment capacity. This study enabled scientific deepening of the similarities and differences of ARDS due to COVID-19 and other causes, in addition to allowing the formation of guidelines regarding initial ventilatory adjustments in patients with ARDS / COVID-19.
\end{abstract}

Keywords: COVID-19; Betacoronavirus; Respiration artificial; Respiratory distress syndrome adult.

\title{
Resumen
}

A fines de 2019, un grupo de casos de neumonía asociados con un nuevo coronavirus de ARN envuelto, se extendió rápidamente por los continentes y se conoció como la pandemia global de COVID-19. Una parte más pequeña de la población no requerirá hospitalización, sin embargo, algunos pacientes pueden desarrollar una forma grave de la enfermedad y presentar complicaciones como un síndrome de dificultad respiratoria aguda (SDRA) que requiere ventilación mecánica invasiva (VMI). Los pacientes con SDRA por COVID-19 pueden presentar algunas características específicas en comparación con las formas típicas del síndrome, desarrollando fenotipos distintos que aún se han estudiado. La investigación se ha centrado en aclarar la ventilación pulmonar protectora (VLP) para estos pacientes. Esta revisión narrativa tuvo como objetivo investigar la experiencia mundial actual en relación con VLP como manejo del SDRA asociado con COVID-19 y resaltar las prácticas de IMV durante una pandemia. Después de un proceso de seguimiento de los estudios en las bases de datos PubMed y BVS, se seleccionaron 20 estudios. En la mayoría de los estudios, el manejo ventilatorio de los pacientes es similar al SDRA "clásico" y, por lo tanto, un VLP se destacó como un aliado importante en la regresión del SDRA en pacientes COVID-19. Sin embargo, se debe tener en cuenta la heterogeneidad fenotípica de los pacientes, especialmente en lo que respecta a la mecánica pulmonar y la capacidad de reclutamiento. Este estudio permitió profundizar científicamente las similitudes y diferencias del SDRA por COVID-19 y otras causas, además de permitir la formación de pautas sobre ajustes ventilatorios iniciales en pacientes con SDRA / COVID-19.

Palabras clave: COVID-19; Betacoronavirus; Respiración artificial; Síndrome de dificultad respiratoria del adulto.

\section{Introdução}

No final de 2019, emergiu em Wuhan, na China, um grupo de casos de pneumonia associados a um novo coronavírus de RNA envelopado (SARS-Cov-2), espalhando-se rapidamente pelos continentes e sendo rotulado pela Organização Mundial da Saúde (OMS) como uma pandemia global por COVID-19 (doença associada ao vírus). Globalmente, em 21 de dezembro de 2020, o surto da COVID-19 resultou no alarmante número de 75.704 .857 casos confirmados, incluindo 1.690 .061 mortes, notificados à OMS (World Health Organization, 2020).

Os pacientes com COVID-19 podem se apresentar em um amplo espectro clínico, desde a respiração normal percebida ("hipoxemia silenciosa") até o comprometimento respiratório com uma ampla variação de recursos sobrepostos entre eles (Gattinoni L et al., 2020a). Uma proporção considerável da população com COVID-19 não necessitará de hospitalização, no entanto, alguns pacientes podem desenvolver a forma grave da doença e apresentar complicações como a síndrome do desconforto respiratório agudo (SDRA) que requer tratamento em unidade de terapia intensiva (UTI) e ventilação mecânica invasiva (VMI) (Yang X et al., 2020). Uma grande série de casos de 1.300 pacientes com COVID-19 da Itália descobriu que $88 \%$ dos pacientes criticamente enfermos necessitaram de VMI (Grasselli et al., 2020). 
Pacientes com pneumonia por COVID-19 que atendem aos critérios de Berlim para SDRA, entretanto, podem apresentar algumas características específicas em comparação com as formas típicas da síndrome, como uma hipoxemia grave contrastando com preservação relativa na mecânica respiratória (Gattinoni et al., 2020b; Liu et al., 2020). Gattinoni et al. (2020b), destacaram a heterogeneidade de pacientes com SDRA e COVID-19 e propuseram a existência de dois fenótipos primários: tipo Low (L) e tipo High (H), com cada um tendo sua via fisiopatológica distinta, sendo o último mais consistente com o que eles descrevem como SDRA típica. Os autores propuseram ainda uma conversão fenotípica que, se não corrigida, pode induzir uma espiral descendente rápida em direção a lesão pulmonar progressiva, vasoplegia e encolhimento pulmonar, com risco de lesão pulmonar induzida por ventilação (VILI) (Gattinoni et al., 2020b). Compreender essas nuances é vital para fornecer tratamento adequado, inclusive no que concerne à VMI.

Diante disso, as pesquisas têm se concentrado em esclarecer estratégias ventilatórias protetoras do pulmão (Slutsky A \& Ranieri M, 2013). As diretrizes práticas da Surviving Sepsis Campaign e National Institutes of Health (NIH) (Alhazzani W et al., 2020) sobre o manejo da COVID-19 recomendaram inicialmente que "pacientes com COVID-19 ventilados mecanicamente devam ser tratados de forma semelhante a outros pacientes com insuficiência respiratória aguda na UTI". De acordo com as Diretrizes Brasileiras de Ventilação Mecânica (Barbas CSV et al., 2014), os ajustes ventilatórios de proteção pulmonar na SDRA incluem: volume corrente (VC) ajustado entre 3 e $6 \mathrm{~mL} / \mathrm{kg}$ de peso corporal previsto; menor fração inspirada de oxigênio (FiO2) possível para garantir saturação periférica de $\mathrm{O} 2(\mathrm{SpO} 2)>92 \%$; frequência respiratória (FR) menor ou igual a 45 ipm, desde que não ocasione auto-PEEP; pressão de platô (Pplat) nas vias aéreas menor ou igual a 30 $\mathrm{cmH}_{2} \mathrm{O}$; driving pressure (DP) $\leq 15 \mathrm{cmH}_{2} \mathrm{O}$; e positive end expiratory pressure (PEEP) $\geq 5 \mathrm{cmH}_{2} \mathrm{O}$. Ademais, Amato et al., (2015) demonstraram que a proteção pulmonar com diminuições no DP, devido a mudanças nas configurações do ventilador, foram mais fortemente associadas ao aumento da sobrevida do que o VC e a PEEP (Amato MBP et al., 2015).

A experiência global no tratamento de pacientes com SDRA associada a COVID-19 está se expandindo rapidamente junto com um número crescente de relatórios sobre o manejo ventilatório (Pan C et al., 2020; Ziehr DR et al., 2020). Concomitante a emergência de produção científica sobre o manejo da ventilação mecânica nesses pacientes, surge a necessidade de um rastreamento desses estudos para a fundamentação de evidências sobre o tema.

Diante disto, o objetivo desta revisão foi investigar a experiência mundial atual em relação à estratégia de ventilação protetora pulmonar (EPP) como manejo da SDRA associada à COVID-19 e destacar considerações para as práticas de VMI durante a pandemia COVID-19.

\section{Metodologia}

O estudo trata-se de uma revisão narrativa da literatura de natureza qualitativa (Pereira, et al., 2018). Sendo assim, o estudo foi orientado pela seguinte pergunta: como tem sido a aplicação da EPP diante do cenário atual de casos graves de SDRA associado à COVID-19?

Foi realizada pesquisa bibliográfica nas bases de dados eletrônicas da Biblioteca Virtual de Saúde (BVS) e PubMed, reconhecendo estudos que abordavam a relação ou procedimento do uso da VMI no tratamento de pacientes com a COVID-19. Para tal, utilizou-se termos de busca nas línguas portuguesa (BVS) e inglesa (PubMed), recuperando-se em uma busca geral o conteúdo de publicações de 2019 a 2020. Foi utilizado o conector booleano "AND" para combinar os termos sugeridos, definida em busca nos "Títulos e Resumos", com filtro de acesso aberto aos artigos. As buscas nas duas bases de dados foram realizadas nos dias 10 e 11 de novembro de 2020 (BVS) e 12 de novembro de 2020 (PubMed) (Tabela 1 e Tabela 2). 
Tabela 1. Termos de busca e número de manuscritos recuperados na base de dados BIREME/BVS.

\begin{tabular}{cc}
\hline Termos de busca & Base de dados \\
\hline COVID “and” Ventilação mecânica invasiva & BIREME/BVS \\
COVID “and” Ventilação mecânica & 901 \\
COVID “and” Ventilação artificial & 862 \\
COVID “and” Respiração artificial & 857 \\
COVID-19 “and” Ventilação mecânica invasiva & 59 \\
COVID-19 “and” Ventilação mecânica & 901 \\
COVID-19 “and” Ventilação artificial & 862 \\
COVID-19 “and” Respiração artificial & 857 \\
SARS-COV2 “and” Ventilação mecânica invasiva & 50 \\
SARS-COV2 “and” Ventilação mecânica & 778 \\
SARS-COV2 “and” Ventilação artificial & 751 \\
SARS-COV2 “and” Respiração artificial & 749 \\
Coronavírus “and” Ventilação mecânica invasiva & 58 \\
Coronavírus “and” Ventilação mecânica & 914 \\
Coronavírus “and” Ventilação artificial & 877 \\
Coronavírus “and” Respiração artificial & 873 \\
\hline
\end{tabular}

BIREME: Biblioteca Regional de Medicina; BVS: Biblioteca Virtual de Saúde; COVID: Corona Virus Disease; COVID-19: Corona Virus Disease 2019; SARS-COV2: Severe Acute Respiratory Syndrome Coronavirus 2.

Fonte: Autores. 
Tabela 2. Termos de busca e número de manuscritos recuperados na base de dados PubMed.

\begin{tabular}{|c|c|}
\hline \multirow{2}{*}{ Termos de busca } & \multirow{2}{*}{$\begin{array}{c}\text { Base de dados } \\
\text { PubMed }\end{array}$} \\
\hline & \\
\hline COVID “and” Invasive mechanical ventilation & 287 \\
\hline COVID "and" Mechanical ventilation & 1186 \\
\hline COVID “and" Artificial ventilation & 6 \\
\hline COVID “and" Artificial respiration & 3 \\
\hline COVID-19 “and” Invasive mechanical ventilation & 287 \\
\hline COVID-19 “and" Mechanical ventilation & 1182 \\
\hline COVID-19 "and" Artificial ventilation & 6 \\
\hline COVID-19 "and" Artificial respiration & 3 \\
\hline SARS-COV2 "and" Invasive mechanical ventilation & 9 \\
\hline SARS-COV2 "and" Mechanical ventilation & 22 \\
\hline SARS-COV2 "and" Artificial ventilation & 1 \\
\hline SARS-COV2 “and" Artificial respiration & 0 \\
\hline Coronavirus “and” Invasive mechanical ventilation & 193 \\
\hline Coronavirus "and" Mechanical ventilation & 795 \\
\hline Coronavirus "and" Artificial ventilation & 4 \\
\hline Coronavirus "and" Artificial respiration & 6 \\
\hline
\end{tabular}

PubMed: Serviço de publicações da U.S. National Library of Medicine; COVID: Corona Virus Disease; COVID-19: Corona Virus Disease 2019; SARS-COV2: Severe Acute Respiratory Syndrome Coronavirus 2. Fonte: Autores.

Foram incluídos nesta revisão documentos que atenderam os seguintes critérios: formato de artigo, com resultados que abordaram a aplicação da VMI em pacientes acometidos com a COVID-19 e que apresentaram os principais parâmetros do protocolo da ventilação para o quadro de insuficiência respiratória gerado pela doença, como: VC, PEEP, FR e FiO2. Foram excluídos artigos de opinião, de revisão, relatos de caso, séries de casos e documentos não completamente disponíveis nas bases de dados ou na internet.

Diante disso, a seleção final foi feita da seguinte forma: inicialmente, (1) houve a exclusão de documentos que não fossem artigos e não correspondessem aos critérios de inclusão exigidos anteriormente, (2) foram excluídos artigos repetidos. Em seguida, foram aceitos artigos potencialmente elegíveis a partir da (3) leitura dos títulos, (4) leitura dos objetivos e (5) leitura dos resultados. Com isso, foi realizado uma síntese qualitativa (narrativa) das evidências encontradas em resposta à pergunta da pesquisa.

\section{Resultados e Discussão}

Através da estratégia inicial de pesquisa que combinou termos apresentados nas Tabelas 1 e 2, foram recuperados 14.398 estudos, sendo 10.408 encontrados na BVS e 3.990 no PubMed, dos quais 12.620 eram duplicados em ambas bases de dados e foram, portanto, excluídos. Foram rastreados 1.778 artigos, sendo que destes, foram excluídos 1.638 após a leitura dos títulos e resumos e 120 após a leitura dos textos completos, restando 20 estudos para análise. O processo de identificação, seleção, elegibilidade e inclusão dos estudos podem ser observados na Figura 1. 
Figura 1. Fluxo de identificação, seleção e inclusão das produções científicas.
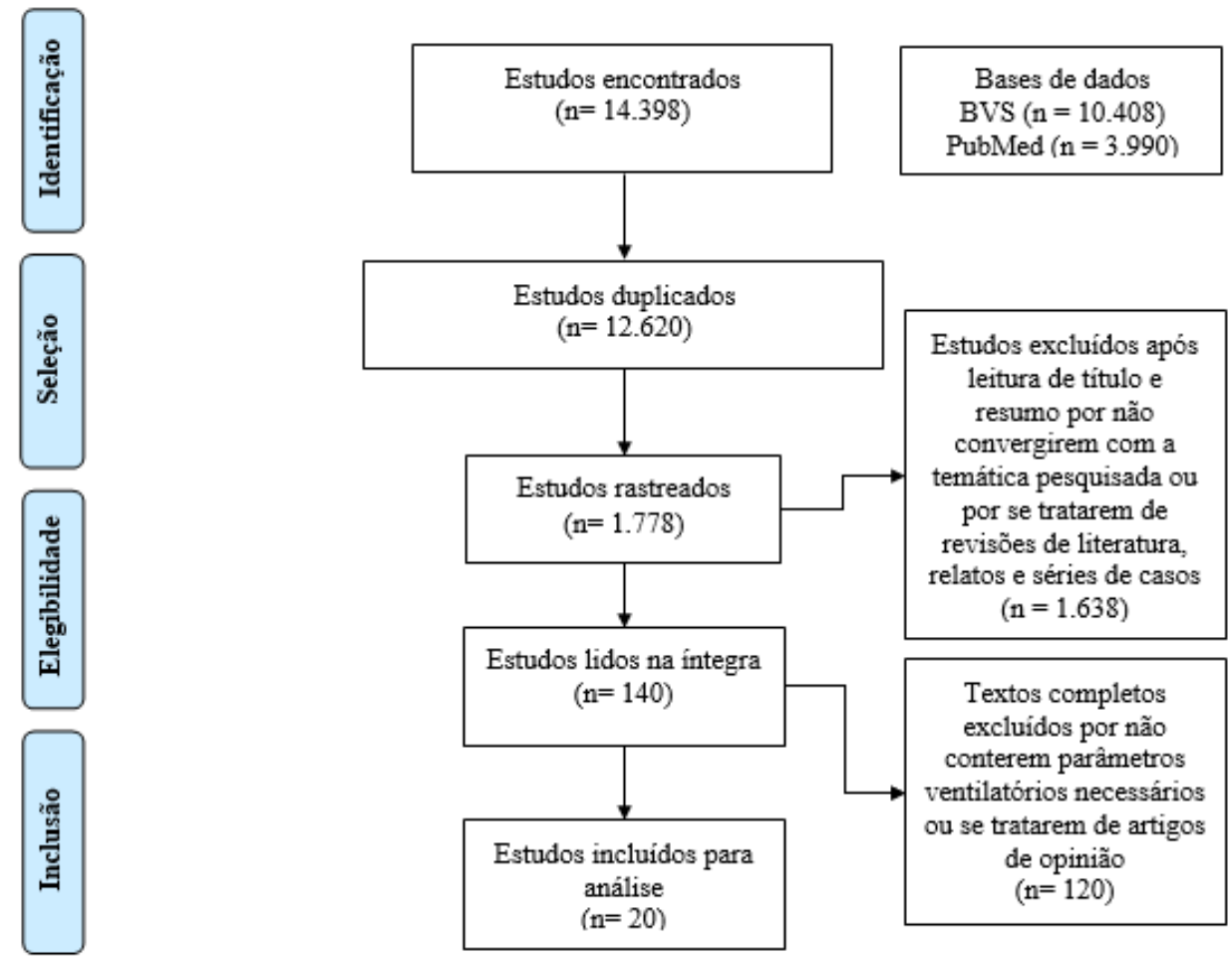

BVS: Biblioteca Virtual de Saúde; PubMed: Serviço de publicações da U.S. National Library of Medicine. Fonte: Autores.

A Tabela 3 descreve uma síntese dos estudos selecionados para esta revisão segundo autor, desenho do estudo, local, faixa etária e número de pacientes em VMI, estratégia de ventilatória e principais desfechos de cada estudo.

Tabela 3. Síntese dos estudos incluídos na revisão segundo autor, desenho do estudo, local, faixa etária e número de pacientes em VMI, estratégia de ventilação e principais desfechos.

\begin{tabular}{|c|c|c|c|c|c|}
\hline Autor & $\begin{array}{l}\text { Desenho de } \\
\text { estudo }\end{array}$ & Local & $\begin{array}{c}\text { Faixa etária e (n) de } \\
\text { pacientes em VMI }\end{array}$ & Estratégia de ventilação & Principais desfechos \\
\hline $\begin{array}{c}\text { Auld SC et } \\
\text { al }\end{array}$ & $\begin{array}{l}\text { Estudo de coorte } \\
\text { observacional } \\
\text { retrospectivo }\end{array}$ & EUA & $\begin{array}{l}\text { Todas as faixas } \\
\text { etárias }(n=165)\end{array}$ & $\begin{array}{c}\mathrm{P} / \mathrm{F}=132 \mathrm{mmHg} / \mathrm{C}_{\mathrm{st}}=34 \\
\mathrm{ml} / \mathrm{cmH}_{2} \mathrm{O} / \mathrm{EPP}\end{array}$ & $\begin{array}{c}\text { Nossa experiência inicial indica que } \\
\text { muitos pacientes sobrevivem à doença } \\
\text { crítica. }\end{array}$ \\
\hline $\begin{array}{l}\text { Cavayas } \\
\text { YA et al }\end{array}$ & $\begin{array}{l}\text { Estudo de coorte } \\
\text { observacional } \\
\text { retrospectivo em } \\
\text { um único centro }\end{array}$ & Canadá & Adultos/ $(n=43)$ & $\begin{array}{c}\mathrm{VC}=7,5 \mathrm{ml} / \mathrm{kg} / \mathrm{PEEP}=9 \\
\mathrm{cmH}_{2} \mathrm{O} / \mathrm{FR}=20 \mathrm{rpm} / \mathrm{FIO}_{2}= \\
50 \% / \mathrm{DP}=13 \mathrm{cmH}_{2} 0 / \text { PPlat }= \\
21 \mathrm{cmH}_{2} \mathrm{O} / \\
\text { Posição prona/ } \mathrm{P} / \mathrm{F}=177 \\
\mathrm{mmHg} / \mathrm{C}_{\mathrm{rs}}=48 \mathrm{~mL} / \mathrm{cmH}_{2} \mathrm{O} / \\
\mathrm{EPP}\end{array}$ & $\begin{array}{l}\text { Características e resultados dos pacientes } \\
\text { foram semelhantes aos relatados na } \\
\text { literatura. A VNI com pressão positiva } \\
\text { teve uma alta taxa de falha quando usada } \\
\text { em pacientes criticamente enfermos. A } \\
\text { lesão vascular pulmonar pode estar no } \\
\text { centro do dano pulmonar induzido por } \\
\text { COVID-19. }\end{array}$ \\
\hline
\end{tabular}




$\begin{array}{cccc}\begin{array}{c}\text { Chao JY et } \\ \text { al }\end{array} & \begin{array}{c}\text { Estudo } \\ \text { retrospectivo }\end{array} & \text { EUA } & \begin{array}{c}\text { Crianças de } 1 \text { mês a } \\ 21 \text { anos }(\mathrm{n}=6)\end{array} \\ & & & \\ \text { Cummings } & \begin{array}{c}\text { Estudo de coorte } \\ \text { observacional } \\ \text { MJ et al }\end{array} & \text { EUA } & \begin{array}{c}\text { Adultos } \geq 18 \text { anos/ } \\ (\mathrm{n}=203)\end{array}\end{array}$

Estudo

Derespina observacional

KR et al retrospectivo

multicêntrico

Evrard B et

al

Não esclarecido

Estudo de coorte

$\begin{array}{cccc}\text { Ferrando C } & \text { prospectivo, } & \text { Espanha e } & \begin{array}{c}\text { Adultos } \geq 18 \text { anos/ } \\ \text { et al }\end{array} \\ & \text { multicêntrico e } & \text { Andorra } & (\mathrm{n}=742) \\ & \text { observacional } & & \end{array}$

Estudo

$\begin{array}{cc}\text { Gamberini } & \text { observacional } \\ \text { L et al } & \text { prospectivo } \\ & \text { multicêntrico }\end{array}$

Itália

Adultos $\geq 18$ anos (n=391)
$\mathrm{VC}=7,8 \mathrm{ml} / \mathrm{kg} / \mathrm{PEEP}=10$ $\mathrm{cmH}_{2} \mathrm{O} / \mathrm{FIO}_{2}=100 \% / \mathrm{P} / \mathrm{F}=$ $178 \mathrm{mmHg}$

$$
\begin{gathered}
\mathrm{VC}=6,2 \mathrm{ml} / \mathrm{kg} / \mathrm{PEEP}=15 \\
\mathrm{cmH}_{2} \mathrm{O} / \mathrm{FR}=22 \mathrm{rpm} / \mathrm{FIO}_{2}= \\
100 \% / \mathrm{DP}=15 \mathrm{cmH}_{2} \mathrm{O} / \\
\text { PPlat }=27 \mathrm{cmH}_{2} \mathrm{O} \\
\text { Posição prona/ } \mathrm{P} / \mathrm{F}=129
\end{gathered}
$$
$\mathrm{mmHg} / \mathrm{C}_{\mathrm{rs}}=27 \mathrm{~mL} / \mathrm{cmH}_{2} \mathrm{O} /$ EPP

$\mathrm{PEEP}=9,5 \mathrm{cmH}_{2} \mathrm{O} / \mathrm{FIO}_{2}=$ $60 \%$

Crianças de 1 mês a $21 \operatorname{anos}(n=20)$

Todas as faixas etárias $(n=18)$

informado Posição prona/ $\mathrm{P} / \mathrm{F}=177$ $\mathrm{mmHg} / \mathrm{EPP}$

Descreveu-se uma taxa mais alta do que a reconhecida anteriormente de doença grave que requer admissão na UTI em pacientes pediátricos internados no hospital com COVID-19.

A doença crítica entre pacientes com COVID-19 na cidade de Nova York é comum e está associada a uma alta frequência de VMI, disfunção orgânica extrapulmonar e mortalidade hospitalar substancial.

Crianças gravemente doentes com COVID-19 são predominantemente adolescentes, têm comorbidades e requerem algum tipo de suporte respiratório. A presença de SDRA está associada a internação prolongada na UTI e hospital.

$\mathrm{VC}=5,2 \mathrm{ml} / \mathrm{kg} / \mathrm{PEEP}=10$ $\mathrm{cmH}_{2} \mathrm{O} / \mathrm{FR}=24 \mathrm{rpm} / \mathrm{DP}=$

\section{$12 \mathrm{cmH}_{2} \mathrm{O} /$}

PPlat $=23 \mathrm{cmH}_{2} \mathrm{O} /$ Posição prona/ $\mathrm{P} / \mathrm{F}=130 \mathrm{mmHg} / \mathrm{C}_{\mathrm{rs}}=$ $38 \mathrm{ml} / \mathrm{cmH}_{2} \mathrm{O}$
$\mathrm{VC}=6,9 \mathrm{ml} / \mathrm{kg} / \mathrm{PEEP}=12$ $\mathrm{cmH}_{2} \mathrm{O} / \mathrm{FR}=24 \mathrm{rpm} /$

$\mathrm{FIO}_{2}=80 \% / \mathrm{DP}=12 \mathrm{cmH}_{2} \mathrm{O} /$ Pplat $=25 \mathrm{cmH}_{2} \mathrm{O}$ Posição prona/ $\mathrm{P} / \mathrm{F}=$ $120 \mathrm{mmHg} / \mathrm{C}_{\mathrm{rs}}=35$ $\mathrm{ml} / \mathrm{cmH}_{2} \mathrm{O} / \mathrm{EPP}$

$\mathrm{VC}=7,1 \mathrm{ml} / \mathrm{kg} / \mathrm{PEEP}=$ $12 \mathrm{cmH}_{2} \mathrm{O} / \mathrm{FIO}_{2}=70 \%$ / PPlat $=25 \mathrm{cmH}_{2} \mathrm{O} /$ Posição prona/ $\mathrm{P} / \mathrm{F}=100$

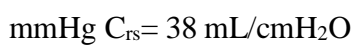

Pacientes com COVID-19 ventilados hemodinamicamente com ETE tem prevalência menor de insuficiência ventricular esquerda e direita do que em pacientes com SDRA relacionada à

gripe.

\section{Pacientes com SDRA/COVID-19} parecem ter características fisiológicas semelhantes a outras causas de SDRA, bem como ajustes ventilatórios, incluindo $\mathrm{C}_{\text {st, }}$ PPlatô e DP. A adesão à EPP foi alta e o risco de mortalidade em 28 dias aumentou com a gravidade da

\section{SDRA.}

Idade, escore SOFA na admissão da UTI, $\mathrm{C}_{\mathrm{rs}}, \mathrm{P} / \mathrm{F}$, complicações renais e cardiovasculares e pneumonia associada a ventilação de início tardio foram fatores de risco independentes para VMI prolongada em pacientes com COVID-

19. 


\begin{tabular}{|c|c|c|c|c|c|}
\hline PDW et al & $\begin{array}{c}\text { observacional } \\
\text { prospectivo }\end{array}$ & & etárias $(\mathrm{n}=137)$ & $\mathrm{P} / \mathrm{F}=136 \mathrm{mmHg}$ & $\begin{array}{l}\text { associada a maior proporção de terapias } \\
\text { de resgate. Creatinina, d-dímero, lactato, } \\
\text { potássio, relação P/F e gradiente alvéolo- } \\
\text { arterial na admissão e cardiopatia } \\
\text { isquêmica são preditores de mortalidade. }\end{array}$ \\
\hline Ge $\mathrm{H}$ et al & $\begin{array}{l}\text { Estudo de coorte } \\
\text { retrospectivo }\end{array}$ & China & $\begin{array}{l}\text { Todas as faixas } \\
\text { etárias }(n=7)\end{array}$ & $\begin{array}{c}\mathrm{VC}=8,2 \mathrm{ml} / \mathrm{kg} / \mathrm{PEEP}=7,92 \\
\mathrm{cmH}_{2} \mathrm{O} / \\
\mathrm{FR}=25,65 \mathrm{rpm} / \text { PPlat }=28,44 \\
\mathrm{cmH}_{2} \mathrm{O} / \\
\text { Posição prona/ } \mathrm{C}_{\mathrm{rs}}=12,28 \\
\mathrm{ml} / \mathrm{cmH}_{2} \mathrm{O}\end{array}$ & $\begin{array}{l}\text { Descreveu-se pela primeira vez a } \\
\text { trajetória completa da mecânica } \\
\text { pulmonar de pacientes com COVID- } \\
\text { 19. Aposição prona foi associada a uma } \\
\text { melhor adesão; PPlat mais alta e uso de } \\
\text { bloqueios neuromusculares associados a } \\
\text { menor risco de IA. }\end{array}$ \\
\hline $\begin{array}{l}\text { Grasselli G } \\
\quad \text { et al }\end{array}$ & $\begin{array}{c}\text { Estudo de coorte } \\
\text { observacional } \\
\text { retrospectivo }\end{array}$ & Itália & $\begin{array}{l}\text { Todas as faixas } \\
\text { etárias }(\mathrm{n}=2929)\end{array}$ & $\begin{array}{c}\mathrm{PEEP}=13 \mathrm{cmH}_{2} \mathrm{O} / \mathrm{FIO}_{2}= \\
70 \% / \mathrm{P} / \mathrm{F}=145 \mathrm{mmHg}\end{array}$ & $\begin{array}{l}\text { A maioria dos pacientes necessitou de } \\
\text { VMI, e a taxa de mortalidade foi alta, } \\
\text { com os seguintes fatores de risco: idade } \\
\text { avançada, sexo masculino, alta FiO2, } \\
\text { PEEP alta ou relação P/F baixa na } \\
\text { admissão na UTI, história de DPOC, } \\
\text { hipercolesterolemia, e diabetes tipo II. }\end{array}$ \\
\hline $\begin{array}{l}\text { Hernandez- } \\
\text { Romieu } \\
\text { AC et al }\end{array}$ & $\begin{array}{l}\text { Estudo de coorte } \\
\text { retrospectivo }\end{array}$ & EUA & $\begin{array}{c}\text { Adultos } \geq 18 \text { anos } \\
\qquad(\mathrm{n}=175)\end{array}$ & $\begin{array}{l}\mathrm{DP}=12 \mathrm{cmH}_{2} \mathrm{O} / \mathrm{P} / \mathrm{F}=148 \\
\mathrm{mmHg} / \mathrm{C}_{\mathrm{st}}=34 \mathrm{ml} / \mathrm{cmH}_{2} 0\end{array}$ & $\begin{array}{l}\text { Nem o tempo desde a admissão na UTI } \\
\text { até a intubação, nem o uso de CNAF } \\
\text { foram associados ao aumento da } \\
\text { mortalidade. A insuficiência respiratória } \\
\text { da COVID-19 pode ser tratada de forma } \\
\text { semelhante à de outras etiologias. }\end{array}$ \\
\hline $\begin{array}{l}\text { Kokoszka- } \\
\text { Bargiel I et } \\
\quad \text { al }\end{array}$ & $\begin{array}{c}\text { Estudo de coorte } \\
\text { observacional } \\
\text { retrospectivo }\end{array}$ & Polônia & Adultos $(n=32)$ & $\begin{array}{c}\mathrm{PEEP}=13,5 \mathrm{cmH}_{2} \mathrm{O} / \mathrm{FIO}_{2}= \\
70 \% / \mathrm{P} / \mathrm{F}=92 \mathrm{mmHg}\end{array}$ & $\begin{array}{l}\text { Os pacientes com COVID-19 que } \\
\text { necessitavam de internação na UTI eram } \\
\text { frágeis e tinham comorbidades } \\
\text { significativas. Os resultados neste grupo } \\
\text { foram ruins e não parecem ser } \\
\text { influenciados pela admissão na UTI. }\end{array}$ \\
\hline $\begin{array}{l}\text { Mauri T et } \\
\text { al }\end{array}$ & $\begin{array}{c}\text { Estudo fisiológico } \\
\text { cruzado } \\
\text { prospectivo }\end{array}$ & Itália & $\begin{array}{c}\text { Adultos } \geq 18 \text { anos } \\
(\mathrm{n}=10)\end{array}$ & $\begin{array}{c}\mathrm{VC}=7,4 \mathrm{ml} / \mathrm{kg} / \mathrm{PEEP}=5 \mathrm{e} \\
15 \mathrm{cmH} \mathrm{H}_{2} \mathrm{O} / \mathrm{FR}=18 \text { a } 25 \\
\mathrm{rpm} / \mathrm{DP}=10 \text { e } 12 \mathrm{cmH}_{2} \mathrm{O} / \\
\mathrm{PPlat}=16 \text { e } 28 \mathrm{cmH}_{2} \mathrm{O} / \mathrm{P} / \mathrm{F}= \\
99 \text { e } 116 \mathrm{mmHg} / \mathrm{C}_{\mathrm{rs}}=47 \mathrm{e} \\
43 \mathrm{ml} / \mathrm{cmH}_{2} \mathrm{O}\end{array}$ & $\begin{array}{c}\text { Em pacientes com SDRA/COVID-19 o } \\
\text { potencial de recrutamento pulmonar } \\
\text { apresenta grande variabilidade, enquanto } \\
\text { a fração de espaço morto elevada pode } \\
\text { ser um traço fisiopatológico } \\
\text { específico. Isso pode orientar a seleção } \\
\text { de configurações personalizadas de } \\
\text { VMI. }\end{array}$ \\
\hline
\end{tabular}




$\begin{array}{cccc}\begin{array}{c}\text { Mittermaie } \\ \text { r M et al }\end{array} & \begin{array}{c}\text { Estudo de coorte } \\ \text { observacional } \\ \text { prospectivo }\end{array} & \text { Alemanha } & \begin{array}{c}\text { Todas as faixas } \\ \text { etárias/ }(\mathrm{n}=15)\end{array} \\ & & \\ & & & \\ \text { Monteiro } & \begin{array}{c}\text { Estudo de coorte } \\ \text { retrospectivo e } \\ \text { ACC et al }\end{array} & \text { EUA } & \text { Todas as faixas } \\ & \text { ebservacional } & & \end{array}$

Primmaz S et al observacional prospectivo
Adultos ( $\mathrm{n}=124)$

$$
\mathrm{VC}=6,4 \mathrm{ml} / \mathrm{kg} / \mathrm{PEEP}=
$$

$18,36 \mathrm{cmH}_{2} \mathrm{O} / \mathrm{FR}=31 \mathrm{rpm} /$

$$
\mathrm{FIO}_{2}=40 \% /
$$

$\mathrm{DP}=9,17 \mathrm{cmH}_{2} \mathrm{O} /$ Posição

prona/ $\mathrm{P} / \mathrm{F}=186,6 \mathrm{mmHg} /$

$\mathrm{C}_{\mathrm{rs}}=59,6 \mathrm{ml} / \mathrm{cmH}_{2} \mathrm{O} / \mathrm{EPP}$

$\mathrm{VC}=6,1 \mathrm{ml} / \mathrm{kg} / \mathrm{PEEP}=5-10$ $\mathrm{cmH}_{2} \mathrm{O} /$ (SDRA leve a moderada); $10-12 \mathrm{cmH}_{2} \mathrm{O}$ (moderada a grave)/ Posição prona/ EPP

$\mathrm{PEEP}=11 \mathrm{cmH}_{2} \mathrm{O} / \mathrm{FR}=32$ $\mathrm{rpm} / \mathrm{FIO} 2=60 \% /$ Posição prona/

$\mathrm{P} / \mathrm{F}=135 \mathrm{mmHg} / \mathrm{EPP}$

$\mathrm{VC}=5,7 \mathrm{ml} / \mathrm{Kg} / \mathrm{PEEP}=10,7$ $\mathrm{cmH}_{2} \mathrm{O}$

Todas as faixas

Tunísia retrospectivo etárias $(n=7)$

Pacientes responderam ao início da VMI com PEEP alta com melhora da oxigenação, e com opacidades pulmonares reduzidas em 6 horas de VMI. Confirma-se a necessidade de PEEP alta para uma oxigenação ideal e DP baixo. A posição prona aumentou a oxigenação.

A mortalidade foi menor do que a relatada. Fatores associados à VMI são obesidade e história de tabagismo. A incidência de SDRA em pacientes intubados é alta.

Antecipação e preparação podem ser responsáveis pela baixa mortalidade observada. Destaca-se a importância da estratégia de gerenciamento de recursos no contexto da pandemia da COVID-19.

Demonstrou-se perfil particular de COVID-19 como uma apresentação grave em homens idosos com comorbidades apresentando um comprometimento neurológico semelhante a SDRA com prognóstico desfavorável.

$\mathrm{VC}=6,1 \mathrm{ml} / \mathrm{kg} / \mathrm{PEEP}=12$ $\mathrm{cm} / \mathrm{H}_{2} \mathrm{O} / \mathrm{FIO}_{2}=72 \% / \mathrm{DP}=13$

$\begin{array}{cccc}\text { Schmidt M } & \text { Estudo de coorte } & \text { França, } & \text { Adultos } \geq 16 \text { anos } \\ \text { et al } & \text { prospectivo } & \text { Bélgica e } & (\mathrm{n}=3376) \\ & \text { multicêntrico } & \text { Suíça } & \end{array}$

Weiss TT

Estudo de coorte

et al observacional retrospectivo

EUA Adultos $(\mathrm{n}=42)$

$$
\begin{gathered}
\mathrm{cmH}_{2} \mathrm{O} / \\
\text { PPlat }=24 \mathrm{cmH}_{2} \mathrm{O} / \text { Posição } \\
\text { prona/ } \mathrm{P} / \mathrm{F}=154 \mathrm{mmHg} / \\
\mathrm{C}_{\mathrm{st}}=33 \mathrm{~mL} / \mathrm{cmH}_{2} \mathrm{O} / \mathrm{EPP} \\
\mathrm{VC}=6,0 \mathrm{ml} / \mathrm{Kg} / \mathrm{PEEP}=15 \\
\mathrm{cmH}_{2} \mathrm{O} / \mathrm{FIO}_{2}=80 \% / \mathrm{PPlat}= \\
27,7 \mathrm{cmH}_{2} \mathrm{O} / \\
\mathrm{P} / \mathrm{F}=134.26 \mathrm{mmHg}^{-} \mathrm{C}_{\mathrm{st}}= \\
33,7 \mathrm{ml} / \mathrm{cmH}_{2} \mathrm{O} / \mathrm{EPP}
\end{gathered}
$$

A mortalidade foi maior em pacientes idosos, diabéticos, obesos e com SDRA grave.

Pacientes com SDRA aguda COVID-19 frequentemente responderam a posição prona inicial com melhora da oxigenação.

N: número; VMI: ventilação mecânica invasiva; VC: volume corrente; PEEP: positive end expiratory positive; FR: frequência respiratória; $F i O_{2}$ : Fração inspirada de oxigênio; DP: driving pressure; Pplat: pressão de platô; P/F: relação pressão parcial de oxigênio para fração inspirada de oxigênio; $\mathrm{C}_{\mathrm{st}}$ : complacência estática; $\mathrm{C}_{\mathrm{rs}}$ : complacência do sistema respiratório; EPP: estratégia de protetora pulmonar; VNI: ventilação não invasiva; COVID-19: Corona Virus Disease 2019; UTI: unidade de terapia intensiva; SDRA: síndrome do desconforto respiratório agudo; ETE: ecocardiografia transesofágica; SOFA: Sequential Organ Failure Assessment; IA: índice de assincronia; DPOC: doença pulmonar obstrutiva crônica ; CNAF: cânula nasal de alto fluxo.

Fonte: Autores. 
Identificou-se oito estudos de caráter prospectivo (Cummings, et al., 2020; Ferrando et al., 2020; Gamberini et al., 2020; Garcia et al., 2020; Mauri et al., 2020; Mittermaier et al., 2020; Primmaz, et al., 2020; Schmidt et al., 2020), onze estudos de caráter retrospectivo (Auld et al., 2020; Cavayas et al., 2020; Chao, et al., 2020; Derespina et al., 2020; Ge et al., 2020; Grasselli et al., 2020; Hernandez-Romieu et al., 2020; Kokoszka-Bargieł et al., 2020; Monteiro et al., 2020; Saida et al., 2020; Weiss TT et al., 2020) e apenas um estudo não teve seu método esclarecido (Evrard, et al., 2020). Nove publicações abrangeram o continente europeu (Ferrando et al., 2020; Gamberini et al., 2020; Grasselli et al., 2020; Hernandez-Romieu et al., 2020; Kokoszka-Bargieł et al., 2020; Mauri et al., 2020; Mittermaier et al., 2020; Primmaz et al., 2020; Schmidt M et al., 2020); oito, o continente americano (Auld et al., 2020; Cavayas et al., 2020; Chao et al., 2020; Cummings et al., 2020; Derespina et al., 2020; Hernandez-Romieu et al., 2020; Monteiro et al., 2020; Weiss et al., 2020); um, o continente asiático (Ge H et al., 2020); um, o continente africano (Saida IB et al., 2020) e um deles não especificou a região de estudo (Evrard et al., 2020). Este achado pode ser explicado pela distribuição global de casos confirmados da COVID-19 relatada pela OMS, que retrata as regiões das Américas e a Europa como mais afetadas, respectivamente.

Ainda sobre os estudos analisados, dez estudos incluíram apenas pacientes adultos (Cavayas et al., 2020; Cummings et al., 2020; Ferrando et al., 2020; Gamberini et al., 2020; Hernandez-Romieu et al., 2020; Kokoszka-Bargieł et al., 2020; Mauri et al., 2020; Primmaz et al., 2020; Schmidt et al., 2020; Weiss et al., 2020), oito estudos incluíram a população em geral (todas as faixas etárias) (Auld et al., 2020; Evrard et al., 2020; Garcia et al., 2020; Ge et al., 2020; Grasselli et al., 2020; Mittermaier et al., 2020; Monteiro et al., 2020; Saida et al., 2020) dois estudos envolveram crianças e adolescentes (Chao, JY et al., 2020; Derespina, et al., 2020). Os artigos relacionados apresentaram uma variação de 6 a 3.376 pacientes em VMI, somando um total de 8.470 pacientes estudados e uma média de 423,5 pacientes por estudo. Os desfechos dos artigos incluídos envolveram principalmente preditores de mortalidade e da doença grave, manejo ventilatório de pacientes na UTI, como o uso da VMI e posição prona, perfil de pacientes e especificidades da SDRA associada à COVID-19.

Ao analisar os estudos encontrados, onze deles abordaram a EPP como diretriz para o tratamento de seus pacientes (Auld et al., 2020; Cavayas et al., 2020; Cummings et al., 2020; Derespina et al., 2020; Ferrando et al., 2020; Mittermaier et al., 2020; Monteiro et al., 2020; Primmaz et al., 2020; 2(8); Saida et al, 2020; Schmidt et al., 2020; Weiss et al., 2020). Quanto aos parâmetros de ventilação abordados, treze estudos apresentaram VC (Cavayas et al., 2020; Chao et al., 2020; Cummings et al., 2020; Evrard et al., 2020; Ferrando et al., 2020; Gamberini et al., 2020; Ge et al., 2020; Mauri et al., 2020; Mittermaier et al., 2020; Monteiro et al., 2020; Saida, et al. 2020; Schmidt et al., 2020; Weiss et al., 2020); dezessete, a PEEP (Cavayas et al., 2020; Chao et al., 2020; Cummings et al., 2020; Derespina et al., 2020; Evrard et al., 2020; Ferrando et al., 2020; Gamberini et al., 2020; Ge et al., 2020; Grasselli et al., 2020; Kokoszka-Bargieł et al., 2020; Mauri et al., 2020; Mittermaier et al., 2020; Monteiro et al., 2020; Primmaz et al., 2020; Saida et al., 2020; Schmidt et al., 2020; Weiss et al., 2020); oito, a FR (Cavayas et al., 2020; Cummings et al., 2020; Ferrando et al., 2020; Ge H et al., 2020; Hernandez-Romieu et al., 2020; Mauri et al.,

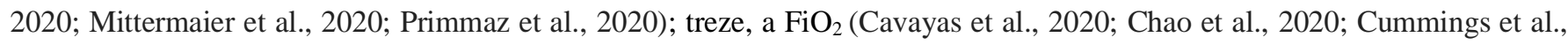
2020; Derespina et al., 2020; Ferrando et al., 2020; Gamberini et al., 2020; Garcia et al., 2020; Grasselli et al., 2020; Kokoszka-Bargieł, et al., 2020: Mittermaier et al., 2020; Primmaz et al., 2020; Schmidt et al., 2020.; Weiss et al., 2020); oito, o DP (Cavayas et al., 2020; Cummings et al., 2020; Evrard et al., 2020; Ferrando et al., 2020; Hernandez-Romieu et al., 2020; Mauri et al., 2020; Mittermaier et al., 2020; Schmidt et al., 2020); dez, a Pplat (Cavayas et al., 2020; Cummings et al., 2020; Evrard et al., 2020; Ferrando et al., 2020; Gamberini et al., 2020; Ge et al., 2020; Mauri et al., 2020; Saida et al., 2020; Schmidt et al., 2020; Weiss , et al., 2020); treze, o uso da posição prona (Cavayas et al., 2020; Cummings et al., 2020; Derespina et al., 2020; Evrard et al., 2020; Ferrando et al., 2020; Gamberini , et al., 2020; Garcia et al., 2020; Ge , et al., 2020; Mittermaier , et al., 2020; Monteiro et al., 2020; Primmaz et al., 2020; Saida et al, 2020; Schmidt et al., 2020); dezoito a relação pressão parcial de $\mathrm{O}_{2}$ para fração inspirada de $\mathrm{O}_{2}$ (P/F) (Auld SC et al., 2020; Cavayas et al., 2020; Chao, et al. 
2020; Cummings et al., 2020; Derespina et al., 2020; Evrard et al., 2020; Ferrando et al., 2020; Gamberini et al., 2020; Garcia et al., 2020; Grasselli et al., 2020; Hernandez-Romieu et al., 2020; Kokoszka-Bargieł et al., 2020; Mauri et al., 2020; Mittermaier et al., 2020; Primmaz et al., 2020; Saida et al., 2020; Schmidt et al., 2020; Weiss , et al., 2020) quatro, a complacência estática $\left(\mathrm{C}_{\mathrm{st}}\right)$ (Auld et al., 2020; Hernandez-Romieu et al., 2020; Schmidt et al., 2020; Weiss , et al., 2020) e oito, a complacência do sistema respiratório $\left(\mathrm{C}_{\mathrm{rs}}\right)$ (Cavayas et al., 2020; Cummings et al., 2020; Evrard et al., 2020; Ferrando et al., 2020; Gamberini et al., 2020; Ge et al., 2020; Mauri et al., 2020; Mittermaier et al., 2020).

Estratégias de ventilação mecânica de proteção que usam pressões de platô baixas, volumes correntes baixos, PEEPs altas e driving pressure menores podem melhorar a sobrevida em pacientes com SDRA (Amato MBP et al., 2015). Em média, os pacientes dos estudos analisados foram tratados com VC baixo e níveis moderados de PEEP dentro do paradigma padrão de EPP na SDRA.

Em relação ao VC aplicado, a diretriz do Surviving Sepsis Campaign para o manejo da COVID-19 recomenda o uso de ventilação com VC baixo (4-8 mL/kg predito) ao contrário de VC mais altos (>8 mL/kg predito) (Alhazzani et al., 2020). Nos estudos analisados, o VC encontrado variou entre 5,2 e $8,2 \mathrm{ml} / \mathrm{kg}$, com uma média de $6,7 \mathrm{ml} / \mathrm{kg}$ do peso corporal predito. Um dos estudos analisados especificou sua adesão à EPP somente como o uso de volume corrente $\mathrm{VC}<8 \mathrm{~mL} / \mathrm{kg}$ de peso predito, destacando sua importância neste sentido (Monteiro ACC, et al., 2020). Este estudo identificou ainda seus pacientes SDRA com um VC médio de $6,1 \mathrm{ml} / \mathrm{kg}$ de peso predito no dia 1 e nos dias 2-7 de 5,9 a 6,1 ml/kg do peso predito ${ }^{28}$. Estes achados indicam que VC menores têm sido utilizados mundialmente como medida de proteção pulmonar para pacientes com SDRA por COVID-19 grave, evitando lesão pulmonar induzida por hiperdistensão alveolar nesses pacientes.

Sabe-se que ventilação com $6 \mathrm{ml} / \mathrm{kg}$ reduz significativamente a mortalidade (9\%) em comparação à ventilação com $12 \mathrm{ml} / \mathrm{kg}$ (De Prost N, et al., 2011). A mecânica do sistema respiratório e o risco de lesão pulmonar em pacientes com SDRA podem piorar rapidamente, especialmente com a retomada dos esforços espontâneos para respirar (Yoshida et al., 2017). Assim, a liberação de altos volumes correntes nesses pacientes pode estar associada a piores desfechos (Fan et al., 2020).

No que concerne à PEEP, no início da pandemia a diretriz Surviving Sepsis Campaign sugeriu ainda uma estratégia de PEEP alta $\left(>10 \mathrm{cmH}_{2} \mathrm{O}\right)$ à uma PEEP baixa, sendo titulada de acordo com a $\mathrm{FiO}_{2}$ para manter uma $\mathrm{SpO}_{2}$ apropriada a fim de prevenir o atelectrauma e, por conseguinte, reduzir a lesão pulmonar induzida por ventilação mecânica (Alhazzani W, et al., 2020). Os valores de PEEP relatada nos estudos variou de 7,9 a 18,3 $\mathrm{cmH}_{2} \mathrm{O}$, com uma média entre os estudos de $11,8 \mathrm{cmH} \mathrm{H}_{2} \mathrm{O}$, indicando que PEEPs moderadas a altas foram utilizadas para ventilar pacientes graves com COVID-19.

Mittermaier et al. (2020), sugeriram que os pacientes com SDRA por COVID-19 inicialmente não diferiam em sua resposta à PEEP alta da SDRA clássica e, portanto, deviam ser ventilados de acordo com os princípios e regimes de SDRA estabelecidos. Esse estudo identificou ainda que níveis elevados de PEEP eram necessários para a melhor oxigenação, em contraste com o estudo de Chao et al. (2020), os quais afirmaram que as EPPs na SDRA foram insuficientes em seus pacientes no terceiro dia, com a necessidade de uma PEEP média de $10 \mathrm{cmH}_{2} \mathrm{O}$. Outro estudo (Grasselli et al., 2020) analisado nesta revisão relatou níveis de PEEP aplicados em seus pacientes na admissão à UTI maiores do que aqueles relatados para o tratamento da SDRA moderada a grave na era pré-COVID-19, sendo necessários para obtenção de uma oxigenação otimizada.

Esta relativa discrepância encontrada nos estudos pode ocorrer devido às diferentes características clínicas e respostas entre os pacientes COVID-19, principalmente no que se refere à recrutabilidade pulmonar. Dados de estudos anteriores sugerem que a mesma PEEP não deve ser aplicada a todos os pacientes com SDRA; e que sua individualização é necessária porque a resposta à PEEP difere com base justamente na mecânica respiratória individual (Fan E et al., 2020). Destaca-se que a PEEP alta pode ser benéfica em pacientes com alta recrutabilidade, mas prejudicial em pacientes com baixa recrutabilidade, podendo levar a efeitos hemodinâmicos adversos ou barotrauma (Fan et al., 2020). 
Ainda conforme a análise dos estudos, os valores de FR dos pacientes esteve entre 20 e 31 rpm, com uma média de 24,8 rpm entre os estudos. Entretanto, nenhum dos onze estudos que abordaram a EPP identificou a FR como uma variável pertinente a essa estratégia. Apesar disso, estudos prévios apresentam que o VC inicial deve ser ajustado com a finalidade de atender a necessidade de ventilação minuto do paciente, desde que a FR inicial seja $\leq 35 \mathrm{rpm}$ (geralmente 14-25 rpm). O motivo para definir este valor relativamente alto é para prevenir a ocorrência de acidose respiratória, que pode ser causada por baixo VC (Turgut, Kars \& Kollu, 2020). Portanto, dada a pouca ênfase ao ajuste da FR nos estudos, atenta-se ao fato que este deve ser um parâmetro melhor avaliado e estudado no contexto da EPP.

Já em relação à $\mathrm{FiO}_{2}$, as diretrizes brasileiras de ventilação mecânica no que se refere a proteção pulmonar da SDRA vigentes recomenda a utilização de menor $\mathrm{FiO}_{2}$ possível para garantir $\mathrm{SpO}_{2}>92 \%$ em todas as categorias de gravidade de SDRA (Barbas CSV, et al., 2014). Sabe-se que altas frações inspiradas de oxigênio podem causar estresse oxidativo, ocasionando efeitos deletérios principalmente ao tecido pulmonar. Nos estudos analisados, os valores da $\mathrm{FiO}_{2}$ esteve entre 40\%-100\%, com média de 70,1\%. No estudo com maior número de pacientes ventilados ( $\mathrm{n}=3.376$ ), a $\mathrm{FiO}_{2}$ mediana foi de $72 \%$ (Auld et al., 2020), revelando certa gravidade no estado clínico dos pacientes, os quais requereram $\mathrm{FiO}_{2}>50 \%$ para manter boa saturação.

Ademais, Grasseli et al. (2020) identificaram que um aumento de $10 \%$ na $\mathrm{FiO}_{2}$ no primeiro dia de admissão está significativamente relacionado ao aumento da mortalidade. Sendo assim, de acordo com conhecimento atual sobre a COVID19, a oxigenação geralmente está comprometida e é necessário uma alta $\mathrm{FiO}_{2}$.

Quanto aos valores de DP observados nos estudos descritos, estes estiveram entre 9,1 e $15 \mathrm{cmH}_{2} \mathrm{O}$, apresentando média de 12,1 $\mathrm{cmH}_{2} \mathrm{O}$. Dois dos estudos concluíram em seus resultados valores de DP muito semelhantes à SDRA não COVID-19 observados em outras pesquisas (Ferrando C, et al., 2020; Schmidt M et al., 2020). Mittermeir et al., (2020) identificaram que níveis elevados de PEEP eram necessários para a oxigenação otimizada, enquanto o DP permanecia baixo, dentro de margens comumente consideradas seguras.

Corroborando com este achado, um estudo anterior relatou uma correlação positiva entre DP>15 $\mathrm{cmH}_{2} \mathrm{O}$ e estresse, tensão e atelectrauma (Pistillo \& Fariña, 2018). Outro estudo ainda revelou que DP alta durante a ventilação de pressão de suporte se associa significativamente com o aumento da mortalidade na UTI (Bellani et al., 2019). Destaca-se, então, a relevância deste parâmetro ventilatório na proteção pulmonar à lesão pulmonar induzida pelo ventilador mecânico, inclusive frente a variáveis como PEEP e VC.

Sobre a pressão de platô utilizada, sua média nos estudos foi de 24,9 $\mathrm{cmH}_{2} \mathrm{O}$, variando entre 21 e 28,4 $\mathrm{cmH}_{2} \mathrm{O}$. Seis dos estudos descreveram a limitação da Pplat como parâmetro apropriado à EPP, geralmente abaixo de $30 \mathrm{cmH}_{2} \mathrm{O}$ (Chao et al., 2020; Cummings et al., 2020; Derespina et al., 2020; Ferrando et al., 2020; Saida et al., 2020; Weiss et al., 2020). Esse achado evidencia que baixos valores de Pplat tem se destacado como um dos principais ajustes necessários para caracterização da EPP.

Uma pesquisa antecedente revelou que a pressão de platô foi um parâmetro ligeiramente melhor do que o DP na previsão de morte hospitalar em pacientes tratados com EPP em 24 horas após o início da SDRA (Villar et al., 2017). No estudo de Ferrando et al. (2020) os valores encontrados para Pplat em pacientes COVID-19 (25 $\mathrm{cmH}_{2} \mathrm{O}$ ) não foram diferentes dos valores obtidos de uma série de grandes estudos observacionais e randomizados relativamente recentes de pacientes com SDRA, fundamentando o princípio de que o manejo ventilatório da SDRA por COVID-19 pouco se diferencia do aplicado à SDRA de outras etiologias. Ademais, outro estudo analisado encontrou como resultado uma associação entre pressão de platô mais alta e menor risco de índice de assincronia do ventilador mecânico e de esforço inspiratório ineficaz durante a expiração (Ge et al., 2020).

A relação P/F nos estudos esteve entre 92 e 186,6 mmHg, apresentando média de 140,3 mmHg. A SDRA foi definida pelos critérios de Berlin com relação P/F 200-300 mmHg como SDRA leve, 100-200 mmHg como SDRA moderada e 
$<100 \mathrm{mmHg}$ como SDRA grave (Force et al., 2012). Os pacientes do estudo de Chao et al., (2020) tinham diagnóstico de hipoxemia moderada a grave de SDRA, com uma relação P/F mediana no dia 1 de $178 \mathrm{mmHg}$ que piorou para $105 \mathrm{mmHg}$ no dia 3, revelando o rápido declínio da função respiratória de alguns pacientes com COVID-19 que podem evoluir aceleradamente para um quadro mais grave.

As relações P/F foram semelhantes entre os estudos de Evrard et al. (2020) (P/F de 130), Auld et al. (2020) (P/F de 132), Weiss et al. (2020) (P/F de 134,26), Primmaz et al. (2020) (P/F de 135) e Garcia et al. (2020) (P/F de 136). Em outro estudo examinado quase todos os pacientes $(96,2 \%)$ tiveram uma relação P/F inferior a 200, valores semelhantes aos da SDRA "clássica" (Gamberini L, et al., 2020). Schimit et al., (2020) relataram que a relação P/F mais baixa é um dos primeiros preditores de mortalidade dos pacientes COVID-19 em 90 dias de internação. Da mesma forma, no estudo de Auld et al., (2020) essa relação foi pior em pacientes que morreram na UTI. Esses dados sugerem que muitos pacientes com COVID-19 em todo o mundo evoluem seu quadro clínico para uma SDRA de moderada a grave, a qual pode estar intimamente relacionada à mortalidade desses pacientes.

Um dos estudos analisados dos EUA encontrou uma grande proporção de pacientes que necessitam de VMI e explicou este fato pela gravidade da hipoxemia, já que o valor da relação P/F encontrado em sua população foi de $129 \mathrm{mmHg}$ (Cummings MJ et al., 2020). Além disso, o estudo de Mittermaier et al. (2020) revelou que após intubação e dentro de 6 horas da VMI, a relação P/F aumentou de 84,3 mmHg para 210,7 mmHg, indicando que a VMI, desde que bem manejada, pode ser forte aliada no tratamento eficaz da SDRA grave causada pela COVID-19.

Considerando as características fisiológicas dos pacientes, quatro dos estudos analisados descreveram a complacência estática dos pacientes, com valores entre 33 e $34 \mathrm{ml} / \mathrm{cmH}_{2} \mathrm{O}$ e, portanto, uma média de 33,6 $\mathrm{ml} / \mathrm{cmH}_{2} \mathrm{O}$. Já a complacência do sistema respiratório foi identificada em oito dos estudos e apresentou valores no intervalo de 27 a 59,6 $\mathrm{ml} / \mathrm{cmH}_{2} \mathrm{O}$ com média de 37,6 ml/ $\mathrm{cmH}_{2} \mathrm{O}$. Na definição de Berlim de SDRA a $\mathrm{C}_{\mathrm{rs}}<40 \mathrm{~mL} / \mathrm{cmH}_{2} \mathrm{O}$ foi anteriormente referida como uma variável auxiliar para definir casos mais graves (Force ADT et al., 2012). Além disso, este parâmetro foi recentemente proposto para identificar um fenótipo mais grave de COVID-19, especialmente importante em termos de recrutabilidade (Gattinoni L et al., 2020a).

Cavayas et al. (2020) indicaram que seus pacientes foram inicialmente caracterizados com $\mathrm{C}_{\mathrm{rs}}$ relativamente preservada ( $48 \mathrm{ml} / \mathrm{cmH}_{2} \mathrm{O}$ ), em contrapartida, Cumings et al. (2020) e Gamberini et al. (2020) descreveram seus pacientes com mecânica pulmonar prejudicada, ou seja, $\mathrm{C}_{\mathrm{rs}}$ reduzida, com valores de $27 \mathrm{ml} / \mathrm{cmH}_{2} \mathrm{O}$ e $38 \mathrm{ml} / \mathrm{cmH}_{2} \mathrm{O}$, respectivamente. Esse resultado heterogêneo fomenta ainda mais a contribuição de Gattinoni et al. (2020), que encontraram que a distinção entre fenótipos COVID-19 era justamente o estado da mecânica pulmonar.

Gamberini et al. (2020) relataram ainda que em seus pacientes, $\mathrm{C}_{\mathrm{rs}}<40 \mathrm{~mL} / \mathrm{cmH}_{2} \mathrm{O}$ foi independentemente associada com ventilação mecânica prolongada e mortalidade, e que, dessa forma, possivelmente representa um pior marcador ventilatório em pacientes com COVID-19. Além disso, outro estudo encontrou complacência estática maior nos sobreviventes do que no grupo de pacientes COVID-19 que não sobreviveu (15,41 vs. 11,19 ml/ $\left.\mathrm{cmH}_{2} \mathrm{O}\right)(\mathrm{Ge} \mathrm{H}$, et al., 2020).

Três dos estudos analisados encontraram $\mathrm{C}_{\mathrm{rs}}$ em pacientes com SDRA da COVID-19 semelhante à encontrada em pacientes com SDRA de outras causas, necessitando de tratamento com níveis relativamente altos de $\mathrm{PEEP}$ e $\mathrm{FiO}_{2}$ para garantir a oxigenação adequada (Ferrando et al., 2020; Gamberini et al., 2020; Hernandez-Romieu et al., 2020). Em contraste, um outro estudo, que comparou pacientes com SDRA induzida por gripe e pacientes com SDRA associada à COVID-19, encontrou que os pacientes do grupo COVID-19 apresentaram $\mathrm{C}_{\mathrm{rs}}$ e relação P/F significativamente maiores do que os pacientes com gripe, mas com valores de VC e PEEP semelhantes entre os grupos (Evrard, et al., 2020). Vale-se salientar, então, que o debate sobre se os pacientes com a COVID-19 possuem características clínicas, fenótipos, mecânica pulmonar e prognósticos distintos da SDRA típica ainda está em andamento. 
Muitos dos estudos analisados descreveram a utilização da posição prona no manejo COVID-19. No estudo de Ferrando et al. (2020) essa manobra foi utilizada em 76\% dos pacientes. Mittermaier et al. (2020) indicaram que a posição prona aumentou substancialmente a oxigenação, revelando uma melhora acentuada na relação P/F na posição prona em comparação com a posição supina. Além disso, sugeriram que os pacientes com SDRA COVID-19 inicial não diferem em sua resposta a prona da SDRA clássica (Mittermaier et al., 2020). Também em comparação com a posição supina, Ge et al. (2020) relataram que a posição prona foi associada a um aumento de $2,31 \mathrm{ml} / \mathrm{cmH}_{2} \mathrm{O}$ na complacência pulmonar.

Entretanto, o impacto dessas manobras de posição prona depende da capacidade de recrutamento do pulmão, que se mostrou variável na SDRA da COVID-19. Gattinoni et al. (2020b) revelaram que o fenótipo "L" da COVID-19 possui baixa recrutabilidade e, portanto, pouco se beneficiaria dessa manobra de posicionamento. Diante disso, destaca-se a importância de uma avaliação clínica para determinação da fenotipagem desses pacientes COVID-19 a fim da fundamentação de um manejo ventilatório mais eficaz nessa população e, portanto, melhores resultados.

No geral, grande parte dos estudos levaram em consideração a EPP como estratégia ventilatória no cuidado aos seus pacientes. Dois desses estudos evidenciaram inclusive uma alta adesão à EPP em suas coortes (Mittermaier et al., 2020; Ferrando et al., 2020).

\section{Considerações Finais}

Diante da emergência de uma acelerada pandemia global por COVID-19, pouco se sabia, ou se sabe, sobre as características clínicas desses pacientes, bem como o correto tratamento, especificamente em termos de VMI para os quadros mais graves de comprometimento pulmonar. Este estudo possibilitou, um aprofundamento cientifico sobre as semelhanças e divergências da SDRA por COVID-19 e por outras causas, assim como sobre as estratégias ventilatórias utilizadas no mundo nesses pacientes.

Observou-se que em grande parte dos estudos analisados, o manejo dos pacientes se assemelhou bastante ao da SDRA "clássica". Entretanto, outros estudos encontraram resultados diferentes. Nesse sentido, evidencia-se a necessidade de cautela na manutenção "cega" de estratégias benéficas comprovadas para SDRA, mas sem evidências para pacientes com SDRA por COVID-19, uma vez que a própria fenotipagem COVID-19 tem se demonstrado heterogênea quanto à mecânica pulmonar e capacidade de recrutamento.

Portanto, sugerimos estudos futuros que possam descrever melhor o fenótipo clínico dos pacientes e associar essa apresentação fenotípica as estratégias ventilatórias utilizadas durante a SDRA causada pela COVID-19, pois só assim podemos esclarecer as vantagens de cada estratégia de ventilação, assim como, as diferenças nos desfechos clínicos para uma estratégia de ventilação mais específica para cada apresentação clínica.

Em conclusão, a EPP abordada nos estudos se mostrou importante aliada na regressão da SDRA nos pacientes COVID-19, apesar de ter sido insuficiente em alguns poucos estudos. Ajustes como VC baixo, PEEP alta, Pplat e DP limitados foram relevantes nos estudos para a abordagem dessa estratégia. Embora mais estudos específicos sejam necessários, a partir dessa revisão fica como orientação que pacientes com SDRA por COVID-19 internados na UTI sejam ventilados inicialmente seguindo estes ajustes, tendo em vista também a individualidade de cada paciente, bem como suas características clinicas e fisiológicas.

\section{Referências}

Alhazzani, W., Møller, M. H., Arabi, Y. M., Loeb, M., Gong, M. N., Fan, E.,. \& Rhodes, A. (2020). Surviving Sepsis Campaign: guidelines on the management of critically ill adults with Coronavirus Disease 2019 (COVID-19). Intensive care medicine, 1-34. https://doi.org/10.1007/s00134-020-06022-5 
Auld, S., Caridi-Scheible, M., Blum, J. M., Robichaux, C. J., Kraft, C. S., Jacob, J. T., \& COVID, E. (2020). ICU and ventilator mortality among critically ill adults with COVID-19. medRxiv. https://doi.org/10.1101/2020.04.23.20076737

Barbas, C. S. V., Ísola, A. M., Farias, A. M. D. C., Cavalcanti, A. B., Gama, A. M. C., Duarte, A. C. M., \& Amado, V. M. (2014). Recomendações brasileiras de ventilação mecânica 2013. Parte I. Revista Brasileira de terapia intensiva, 26(2), 89-121. https://doi.org/10.5935/0103-507X.20140017

Cavayas, Y. A., Noël, A., Brunette, V., Williamson, D., Frenette, A. J., Arsenault, C., \& Albert, M. (2020). Early experience with critically ill patients with COVID-19 in Montreal. Canadian Journal of Anesthesia/Journal canadien d'anesthésie, 1-10. https://doi.org/10.1007/s12630-020-01816-z

Chao, J. Y., Derespina, K. R., Herold, B. C., Goldman, D. L., Aldrich, M., Weingarten, J., \& Medar, S. S. (2020). Clinical characteristics and outcomes of hospitalized and critically ill children and adolescents with coronavirus disease 2019 (COVID-19) at a Tertiary Care Medical Center in New York City. The Journal of Pediatrics. https://doi.org/10.1016/j.jpeds.2020.05.006

Cummings, M. J., Baldwin, M. R., Abrams, D., Jacobson, S. D., Meyer, B. J., Balough, E. M., \& O'Donnell, M. R. (2020). Epidemiology, clinical course, and outcomes of critically ill adults with COVID-19 in New York City: a prospective cohort study. The Lancet. https://doi.org/10.1016/S0140-6736(20)31189-2

De Prost, N., Ricard, J. D., Saumon, G., \& Dreyfuss, D. (2011). Ventilator-induced lung injury: historical perspectives and clinical implications. Annals of intensive care, 1(1), 1-15. https://doi.org/10.1186/2110-5820-1-28

Derespina, K. R., Kaushik, S., Plichta, A., Conway Jr, E. E., Bercow, A., Choi, J., \& Medar, S. S. (2020). Clinical manifestations and outcomes of critically ill children and adolescents with coronavirus disease 2019 in New York city. The Journal of pediatrics, 226, 55-63. https://doi.org/10.1016/j.jpeds.2020.07.039

Evrard, B., Goudelin, M., Montmagnon, N., Fedou, A. L., Lafon, T., \& Vignon, P. (2020). Cardiovascular phenotypes in ventilated patients with COVID-19 acute respiratory distress syndrome. Critical Care, 24(1), 1-5. https://doi.org/10.1186/s13054-020-02958-8

Fan, E., Beitler, J. R., Brochard, L., Calfee, C. S., Ferguson, N. D., Slutsky, A. S., \& Brodie, D. (2020). COVID-19-associated acute respiratory distress syndrome: is a different approach to management warranted?. The Lancet Respiratory Medicine. https://doi.org/10.1016/S2213-2600(20)30304-0

Ferrando, C., Suarez-Sipmann, F., Mellado-Artigas, R., Hernández, M., Gea, A., Arruti, E., \& Villar, J. (2020). Clinical features, ventilatory management, and outcome of ARDS caused by COVID-19 are similar to other causes of ARDS. Intensive care medicine, 46(12), 2200-2211. https://doi.org/10.1007/s00134$020-06192-2$

Gamberini, L., Tonetti, T., Spadaro, S., Zani, G., Mazzoli, C. A., Capozzi, C., \& Ranieri, V. M. (2020). Factors influencing liberation from mechanical ventilation in coronavirus disease 2019: multicenter observational study in fifteen Italian ICUs. Journal of intensive care, 8(1), 1-12. https://doi.org/10.1186/s40560-020-00499-4

Garcia, P. D. W., Fumeaux, T., Guerci, P., Heuberger, D. M., Montomoli, J., Roche-Campo, F., \& RISC-19-ICU Investigators. (2020). Prognostic factors associated with mortality risk and disease progression in 639 critically ill patients with COVID-19 in Europe: Initial report of the international RISC-19-ICU prospective observational cohort. EClinicalMedicine, 25, 100449. https://doi.org/10.1016/j.eclinm.2020.100449

Gattinoni, L., Chiumello, D., Caironi, P., Busana, M., Romitti, F., Brazzi, L., \& Camporota, L. (2020). COVID-19 pneumonia: different respiratory treatments for different phenotypes?. https://doi.org/10.1007/s00134-020-06033-2

Gattinoni, L., Coppola, S., Cressoni, M., Busana, M., Rossi, S., \& Chiumello, D. (2020). Covid-19 does not lead to a "typical" acute respiratory distress syndrome. American journal of respiratory and critical care medicine, 201(10), 1299-1300. https://doi.org/10.1164/rccm.202003-0817LE

Ge, H., Pan, Q., Zhou, Y., Xu, P., Zhang, L., Zhang, J., \& Zhang, Z. (2020). Lung Mechanics of Mechanically Ventilated Patients With COVID-19: Analytics With High-Granularity Ventilator Waveform Data. Frontiers in medicine, 7, 541. https://doi.org/10.3389/fmed.2020.00541

Grasselli, G., Greco, M., Zanella, A., Albano, G., Antonelli, M., Bellani, G., \& Cecconi, M. (2020). Risk factors associated with mortality among patients with COVID-19 in intensive care units in Lombardy, Italy. JAMA internal medicine, 180(10), 1345-1355. https://doi.org/10.1001/jamainternmed.2020.3539

Grasselli, G., Zangrillo, A., Zanella, A., Antonelli, M., Cabrini, L., Castelli, A., \& Pesenti, A. (2020). Baseline characteristics and outcomes of 1591 patients infected with SARS-CoV-2 admitted to ICUs of the Lombardy Region, Italy. Jama, 323(16), 1574-1581. https://doi.org/10.1001/jama.2020.5394

Hernandez-Romieu, A. C., Adelman, M. W., Hockstein, M. A., Robichaux, C. J., Edwards, J. A., Fazio, J. C., \& Auld, S. C. (2020). Timing of intubation and mortality among critically ill coronavirus disease 2019 patients: a single-center cohort study. Critical care medicine. https:// doi.org/10.1097/CCM.0000000000004600

Kokoszka-Bargieł, I., Cyprys, P., Rutkowska, K., Madowicz, J., \& Knapik, P. (2020). Intensive Care Unit Admissions During the First 3 Months of the COVID-19 Pandemic in Poland: A Single-Center, Cross-Sectional Study. Medical science monitor: international medical journal of experimental and clinical research, 26, e926974-1. https:// doi.org/10.12659/MSM.926974

Liu, X., Liu, X., Xu, Y., Xu, Z., Huang, Y., Chen, S., \& Li, Y. (2020). Ventilatory Ratio in Hypercapnic Mechanically Ventilated Patients with COVID-19associated Acute Respiratory Distress Syndrome. American journal of respiratory and critical care medicine,201(10), 1297-1299. https://doi.org/10.1164/rccm.202002-0373LE

Mauri, T., Spinelli, E., Scotti, E., Colussi, G., Basile, M. C., Crotti, S., \& Pesenti, A. (2020). Potential for lung recruitment and ventilation-perfusion mismatch in patients with the acute respiratory distress syndrome from coronavirus disease 2019. Critical Care Medicine. https://doi.org/10.1097/CCM.0000000000004386

Mittermaier, M., Pickerodt, P., Kurth, F., de Jarcy, L. B., Uhrig, A., Garcia, C., \& Müller-Redetzky, H. (2020). Evaluation of PEEP and prone positioning in early COVID-19 ARDS. EClinicalMedicine, 28, 100579. https://doi.org/10.1016/j.eclinm.2020.100579 
Monteiro, A. C., Suri, R., Emeruwa, I. O., Stretch, R. J., Cortes-Lopez, R. Y., Sherman, A., \& Qadir, N. (2020). Obesity and smoking as risk factors for invasive mechanical ventilation in COVID-19: a retrospective, observational cohort study. PloS one, 15(12), e238552. https://doi.org/10.1371/journal.pone.0238552

Pan, C., Chen, L., Lu, C., Zhang, W., Xia, J. A., Sklar, M. C., \& Qiu, H. (2020). Lung Recruitability in COVID-19-associated Acute Respiratory Distress Syndrome: A Single-Center Observational Study.American journal of respiratory and critical care medicine,201(10), 1294-1297. https://doi.org/10.1164/rccm.202003-0527LE

Pereira, A. S., Shitsuka, D. M., Parreira, F. J., Shitsuka, R. 2018. Metodologia da pesquisa científica [e-book]. Santa Maria, RS. 1 Ed. UAB/NTE/UFSM

Pistillo, N., \& Fariña, O. (2018). Driving airway and transpulmonary pressure are correlated to VILI determinants during controlled ventilation. Intensive care medicine, 44(5), 674-675. https://doi.org/10.1007/s00134-018-5092-1

Primmaz, S., Le Terrier, C., Suh, N., Ventura, F., Boroli, F., Bendjelid, K., \& Pugin, J. (2020). Preparedness and reorganization of care for coronavirus disease 2019 patients in a swiss ICU: characteristics and outcomes of 129 patients. Critical care explorations, 2(8). https://doi.org/10.1097/CCE.0000000000000173

Saida, I. B., Ennouri, E., Nachi, R., Meddeb, K., Mahmoud, J., Thabet, N., \& Boussarsar, M. (2020). Very severe COVID-19 in the critically ill in Tunisia. The Pan African Medical Journal, 35(Suppl 2). https://doi.org/10.11604/pamj.supp.2020.35.136.24753

Schmidt, M., Hajage, D., Demoule, A., Pham, T., Combes, A., Dres, M., \& Grégoire, C. (2020). Clinical characteristics and day-90 outcomes of 4244 critically ill adults with COVID-19: a prospective cohort study. Intensive Care Medicine. https://doi.org/10.1007/s00134-020-06294-x

Slutsky, A. S., \& Ranieri, V. M. (2014). Ventilator-induced lung injury. The New England journal of medicine,370(10), 980-980. https://doi.org/10.1056/nejmc1400293

Turgut, T. E. K. E., KARS, T. U., \& KOLLU, K. Ventilatory Management Strategies For Acute Respiratory Distress Syndrome (Ards) Due To Covid-19 Disease. Eurasian Journal of Critical Care, 2(2), 171-182.

Weiss, T. T., Cerda, F., Scott, J. B., Kaur, R., Sungurlu, S., Mirza, S. H., \& Li, J. (2020). Prone positioning for patients intubated for severe acute respiratory distress syndrome (ARDS) secondary to COVID-19: a retrospective observational cohort study. British journal of anaesthesia. https://doi.org/10.1016/j.bja.2020.09.042

World Health Organization. (2020). WHO Coronavirus Disease (COVID-19) Dashboard. https://covid19.who.int/

Yang, X., Yu, Y., Xu, J., Shu, H., Liu, H., Wu, Y., \& Shang, Y. (2020). Clinical course and outcomes of critically ill patients with SARS-CoV-2 pneumonia in Wuhan, China: a single-centered, retrospective, observational study. The Lancet Respiratory Medicine. https://doi.org/10.1016/S2213-2600(20)30079-5

Yoshida, T., Fujino, Y., Amato, M. B., \& Kavanagh, B. P. (2017). Fifty years of research in ARDS. Spontaneous breathing during mechanical ventilation. Risks, mechanisms, and management. American journal of respiratory and critical care medicine, 195(8), 985-992. https://doi.org/10.1164/rccm.201604$0748 \mathrm{CP}$

Ziehr, D. R., Alladina, J., Petri, C. R., Maley, J. H., Moskowitz, A., Medoff, B. D., \& Hardin, C. C. (2020). Respiratory pathophysiology of mechanically ventilated patients with COVID-19: a cohort study. American Journal of Respiratory and Critical Care Medicine, (ja). https://doi.org/10.1164/rccm.202004$1163 \mathrm{LE}$

Bellani, G., Grassi, A., Sosio, S., Gatti, S., Kavanagh, B. P., Pesenti, A., \& Foti, G. (2019). Driving pressure is associated with outcome during assisted ventilation in acute respiratory distress syndrome. Anesthesiology: The Journal of the American Society of Anesthesiologists, 131(3), 594-604. https://doi.org/10.1097/ALN.0000000000002846

Villar, J., Martín-Rodríguez, C., Domínguez-Berrot, A. M., Fernández, L., Ferrando, C., Soler, J. A., \& Kacmarek, R. M. (2017). A quantile analysis of plateau and driving pressures: effects on mortality in patients with acute respiratory distress syndrome receiving lung-protective ventilation. Critical care medicine, 45(5), 843-850. https://doi.org/10.1097/CCM.0000000000002330

Force, A. D. T., Ranieri, V. M., Rubenfeld, G. D., Thompson, B. T., Ferguson, N. D., \& Caldwell, E. (2012). Acute respiratory distress syndrome. Jama, 307(23), 2526-2533. https://doi.org/10.1001/jama.2012.5669 